INEEL/CON-99-00406

PREPRINT

\title{
Fabry-Perot Laser Ultrasonic Elastic Anisotropy Measurements on a Moving Paper Web
}

\author{
J. B. Walter \\ K. L. Telschow \\ J. P. Gerhardstein \\ B. M. Pufahl \\ C. C. Habeger \\ E. M. Lafond \\ P. H. Brodeur
}

July 25, 1999 - July 30, 1999

\section{$26^{\text {th }}$ Annual Review of Progress in Quantitative Nondestructive Evaluation}

This is a preprint of a paper intended for publication in a journal or proceedings. Since changes may be made before publication, this preprint should not be cited or reproduced without permission of the author.

This document was prepared as a account of work sponsored by an agency of the United States Government. Neither the United States Government nor any agency thereof, or any of their employees, makes any warranty, expressed or implied, or assumes any legal liability or responsibility for any third party's use, or the results of such use, of any information, apparatus, product or process disclosed in this report, or represents that its use by such third party would not infringe privately owned rights. The views expressed in this paper are not necessarily those of the U.S. Government or the 


\title{
FABRY-PEROT LASER ULTRASONIC ELASTIC ANISOTROPY MEASUREMENTS
}

\author{
ON A MOVING PAPER WEB
}

\author{
J. B. Walter and K.L. Telschow \\ Idaho National Engineering and Environmental Laboratory \\ Lockheed Martin Idaho Technologies Co. \\ Idaho Falls, ID 83415-2209 \\ J.P. Gerhardstein, B.M. Pufahl, C. C. Habeger, E.M. Lafond, and \\ P.H. Brodeur \\ Institute of Paper Science and Technology \\ 500 10th St. NW, Atlanta, GA 30318
}

\section{INTRODUCTION}

On-line measurement of material properties is a goal of many manufacturers to improve production and quality. The elastic stiffness of paper is important for the paper industry. Currently, the elastic constants of paper are measured offline with contact ultrasonic methods [1-4]. Piezoelectric transducers are placed in contact with the paper surface to generate and detect plate wave modes, known as Lamb wave modes [5-7]. At low frequencies, where the wavelength of the elastic wave is larger than the paper thickness, two wave modes dominate in the paper, an anti-symmetric or flexural mode and a symmetric or thickness mode. Measurements of the phase velocities of these modes along both the machine direction (MD) and the perpendicular cross direction (CD) of the paper web provide an important parameter revealing the increased stiffness in the paper along the MD direction.

Laser ultrasonic techniques offer the possibility of making these measurements online, while the paper is moving on the paper web, since physical contact with the paper is eliminated. These techniques both generate and detect elastic wave motion with lasers. Generation is provided by a pulsed laser, which delivers a very short but high intensity impulse to the paper through absorption of the light at the surface. Either thermoelastic expansion, at low energies, or ablation of material, at high energies, convert the energy impulse into elastic waves in paper. The first is non-damaging and preferred for paper but is much less efficient, producing smaller wave motions. An optical interferometer is used to demodulate the small phase modulation that the elastic wave produces on a $\mathrm{CW}$ probe laser beam reflected from the detection spot.

Ultrasonic motion of a surface can be measured with many optical techniques [8]. These methods are based on optical interferometry and have similar sensitivities. The 
detection system signal-to-noise ratio, in the shot noise limited regime, is controlled by the amount of light entering the interferometer. Because diffuse surfaces scatter incident light into a hemisphere, only a very small portion of the incident light contributes to the detected signal. For a standoff distance of a few centimeters, optical collection efficiencies are typically in the $0.1 \%-1 \%$ range. There is also a limit to how much light can be focussed onto the paper without damaging it, the limit depending on the paper, the wavelength of the light and the size of the detection spot. For detection techniques that can use the light from multiple speckles, a larger detection spot can be used.

A confocal Fabry-Perot interferometer is particularly suited to this situation [9]. The technique is self-referencing in that the photodiode signal results from the interference of light scattered from the paper surface and delayed portions of that light generated from multiple reflections within the Fabry-Perot cavity. As all of the optical wave fronts are nearly identical to that scattered directly from the paper surface, light from a relatively large surface area (1-4 $\left.\mathrm{mm}^{2}\right)$ including many speckles is collected and interfered efficiently. This gives the Fabry-Perot technique the optical efficiency needed to provide sufficient sensitivity for detection from diffuse material surfaces such as those of most types of paper without utilizing light levels that damage the paper.

Measurements off-line, on stationary paper, have been successfully made using a Michelson interferometer [10-12]. Motion of the paper surface, such as on-line during paper manufacture on a paper web, introduces additional problems. Laser ultrasonics offers great potential for implementation in the field, and even in situ during the papermaking process. This paper describes the challenges of applying laser ultrasonics to a moving surface and reports on successful measurements on a moving sheet of paper using a Fabry-Perot detector.

\section{MEASUREMENTS ON STATIONARY PAPER}

Initial measurements were made on stationary samples. The experiment is depicted in Figure 1. The incident detection light, an argon ion laser capable of providing 1.5 watts at $514 \mathrm{~nm}$, was focussed onto the surface with optics not shown. Scattered light was collected by lens L1, irrespective to polarization, and processed by the Fabry-Perot interferometer. Generation was provided by a pulsed Nd:YAG laser with a pulse width of about $15 \mathrm{~ns}$ and pulse energy up to $400 \mathrm{~mJ}$ at $1064 \mathrm{~nm}$. The generation pulse was focussed onto the surface by lens L2, which was either a cylindrical lens for a line focus, or a spherical lens for a spot focus.

Figure 2a presents the lowest order symmetric $\left(\mathrm{S}_{0}\right)$ and antisymmetric $\left(\mathrm{A}_{0}\right)$ waveforms on a stationary $0.1 \mathrm{~mm}$ thick stainless steel sheet. It is evident that the uniform line source employed produced little or no signal between the $S_{0}$ wave at $2.5 \mu$ s and the onset of the $\mathrm{A}_{0}$ wave at $3.5 \mu \mathrm{s}$. Figure $2 \mathrm{~b}$ shows equivalent waveforms recorded for

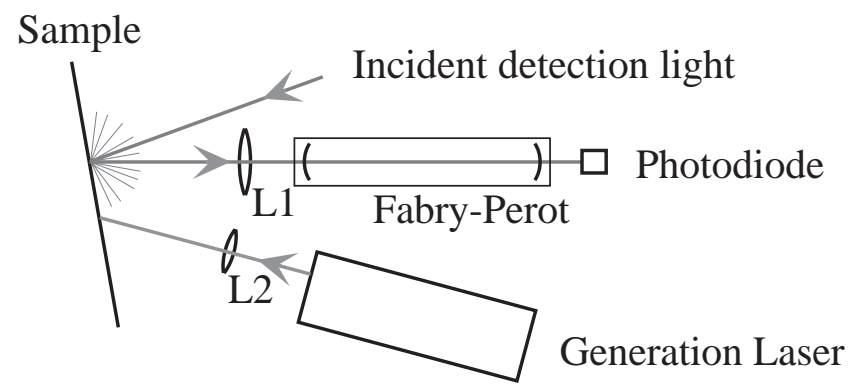

Figure 1. Experimental arrangement for Lamb wave mode measurements on a stationary sheet. 
$0.1 \mathrm{~mm}$ copier paper, commercial paper used in photocopiers. Taking note of the longer time scale for Figure $2 b$, it can be seen that the general frequency content is lower in paper than for stainless steel. This is due to larger attenuation of the Lamb waves in paper. Also evident is the complex signal from the $S_{0}$ wave, extending from about $3 \mu$ s until the onset of the $A_{0}$ wave at $15 \mu \mathrm{s}$. This is believed to be due to the heterogeneity of the paper at the fiber level, resulting in non-uniform generation along the generation line and also scattering of the wave between generation and detection.

Measurements of the $S_{0}$ and $A_{0}$ mode wave velocities were taken as a function of propagation direction on 59\# raw stock paper. Single shot waveforms were recorded with slight ablation, using a spot focus about $6 \mathrm{~mm}$ from the detection spot. The paper was rotated keeping the source and detector geometry fixed. Wave speeds were determined from the earliest signal arrival time. The $S_{0}$ wave velocity in the MD was $3.75 \mathrm{~km} / \mathrm{s}$ as determined by measurements taken over multiple distances for a signal with a bandwidth of

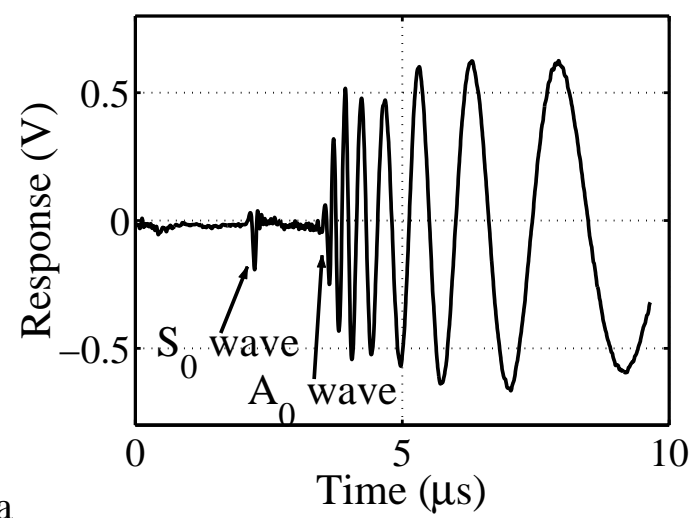

a

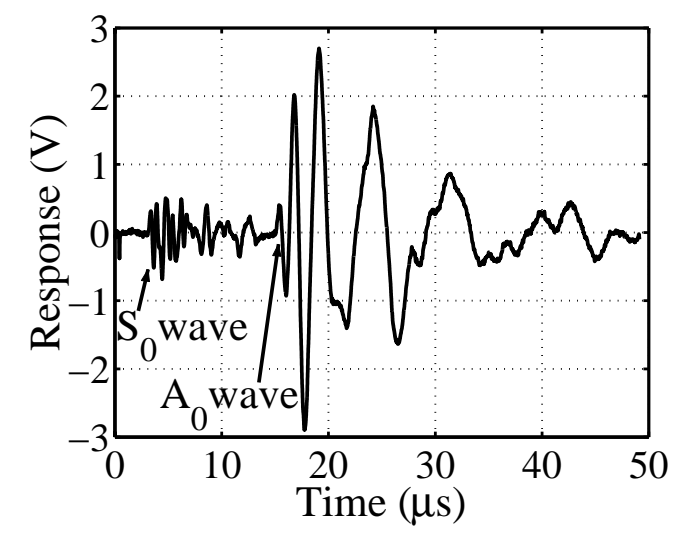

Figure 2. Lowest order Lamb waves generated with a line source and measured with a Fabry-Perot detector for a) $0.1 \mathrm{~mm}$ thick stainless steel and b) $0.1 \mathrm{~mm}$ thick copier paper. The waveforms are clearly separated in stainless steel, with little or no signal between the $\mathrm{S}_{0}$ and $\mathrm{A}_{0}$ waveforms.

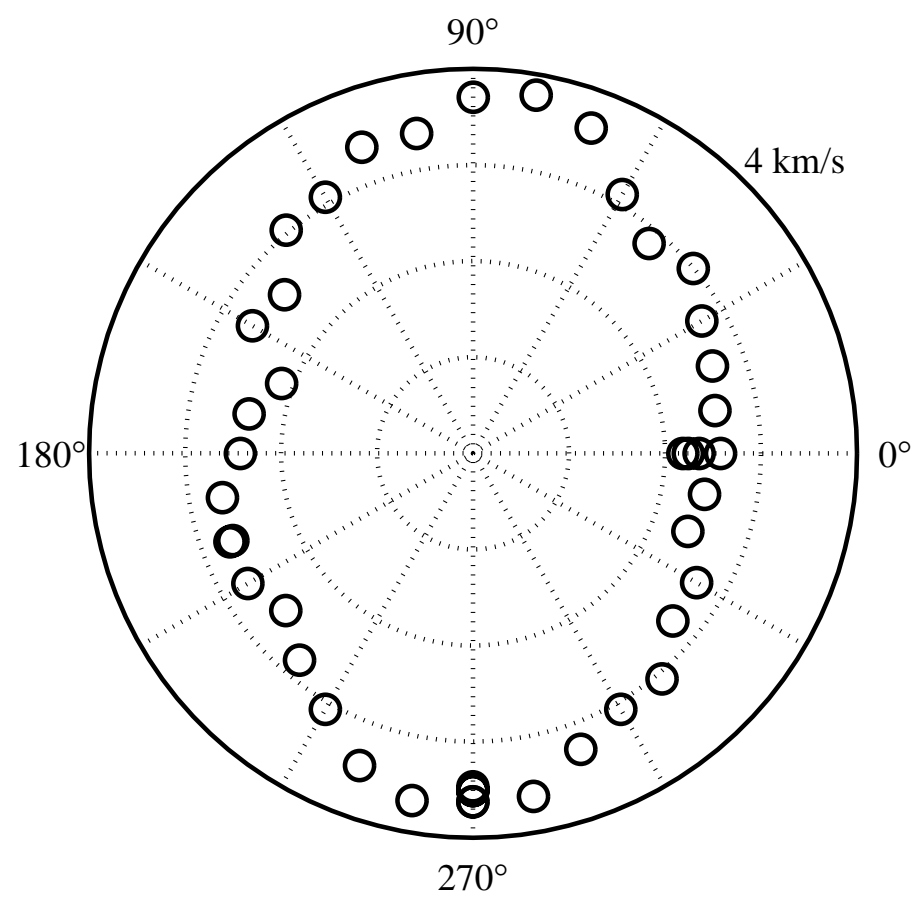

Figure 3. The speed of the $S_{0}$ waveform for a sample of 59\# raw stock paper at varying angles. The machine direction (MD) corresponds to $90^{\circ}$ and $270^{\circ}$. The velocity ratio between the MD and the perpendicular cross direction (CD) was $1.48 \pm .11$ at $1 \mathrm{MHz}$ which agrees well with contact measurement results for this paper. 
about $1 \mathrm{MHz}$. The recorded velocity ratio between the MD and the CD was $1.48 \pm .11$ at $1 \mathrm{MHz}$ which agrees with the contact piezoelectric result $1.41 \pm 0.02$ for this paper [13].

This demonstrated that the Fabry-Perot detector is useful for detection on paper. It effectively accommodated the diffusely reflecting surface of paper, was readily scanned over the paper surface to make measurements at different locations and in different directions, and automatically eliminated low frequency interfering signals due to vibration and laser phase noise. The frequency response of the detector at the Idaho National Engineering and Environmental Laboratory, optimized for operation above $1 \mathrm{MHz}$, is not ideal for measurements on paper. Attenuation in the paper limits the primary wave components in the $A_{0}$ wave to below about $1 \mathrm{MHz}$, while the $S_{0}$ mode shows dispersion above $1 \mathrm{MHz}$. Both of these facts contributed to the complex waveforms recorded in paper.

\section{MEASUREMENTS ON MOVING PAPER}

A machine designed and constructed at the Institute for Paper Science and Technology provided simulation of a moving paper web. It was capable of moving a strip of paper attached to a revolving wheel at speeds up to 400 meters $/$ minute $(\mathrm{m} / \mathrm{min})$, about $25 \%$ of the speed of paper during manufacture. The simulator allowed for contact free optical access to the moving surface.

\section{Velocity Dependent Noise}

Initial measurements on moving paper revealed significant additional noise in the detection process. This noise was due to phase fluctuations that appeared to derive from several sources. Shot to shot variation resulted from fine scale heterogeneity. Local variation in the optical reflectivity produced deviations in both the generation and detection processes, contributing to fluctuations in the strength of the $S_{0}$ and $A_{0}$ modes from one location to another.

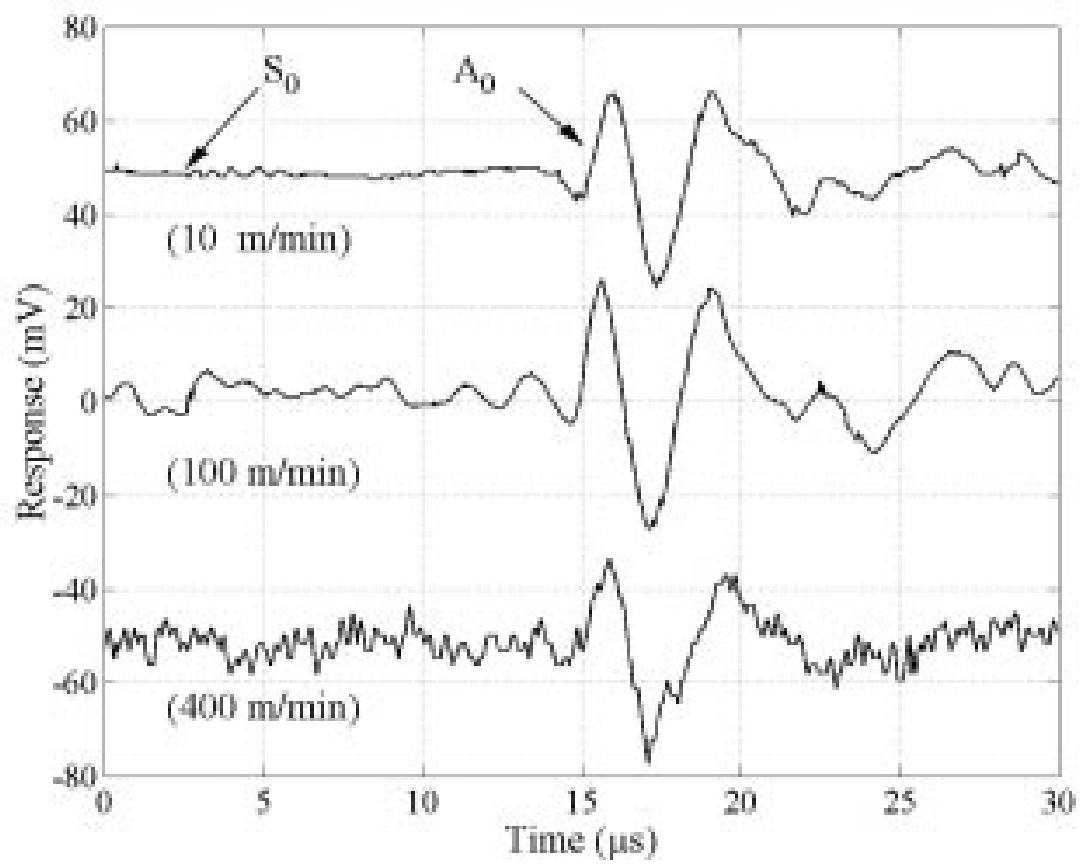

Figure 4. Average waveforms for 42\# liner board at 10, 100 and $400 \mathrm{~m} / \mathrm{min}$. Averaging reveals the $A_{0}$ mode, but at speeds faster than about $10 \mathrm{~m} / \mathrm{min}$ the motion noise is too large for the weaker $\mathrm{S}_{0}$ mode. 
The motion noise can be suppressed by averaging. Figure 4 displays waveforms averaged 128 times for $42 \#$ liner board moving at 10,100 and $400 \mathrm{~m} / \mathrm{min}$. The $A_{0}$ mode is clearly seen even at $400 \mathrm{~m} / \mathrm{min}$, but the $S_{0}$ mode is not discernable at speeds faster than about $10 \mathrm{~m} / \mathrm{min}$ due to the large amplitude of the motion noise. The noise can also be reduced by high pass filtering the signal, but this filters out much of the $\mathrm{A}_{0}$ mode also, and extends the upper speed for the $S_{0}$ mode to only about $200 \mathrm{~m} / \mathrm{min}$

The noise due to motion was examined more closely by recording the Fabry-Perot signal without generating the wave modes with the pulsed laser. Figure 5 presents noise spectra at various speeds for three cases. Figures $5 \mathrm{a}$ and $5 \mathrm{~b}$ show data for $0.04 \mathrm{~mm}$ and $0.26 \mathrm{~mm}$ detection spots, respectively, on 59\# raw stock paper, while Figure 5c shows data for $0.06 \mathrm{~mm}$ detection spot on a relatively smooth surface, the plastic wheel of the simulator. Each contains a large low frequency component that is present even when the simulator is not moving and increases with the simulator speed. Each also contains a plateau noise component that increases in bandwidth but not amplitude as the simulator speed is increased. For a non-specular surface, increasing the spot size reduces the bandwidth of the plateau component. Also, reducing the number of speckles, from the many speckles of the paper to just a few speckles from the plastic wheel, significantly reduces the amplitude of the plateau component. The low frequency component was largely unaffected by either change.

The noise observed includes contributions from several sources. The noise present for a stationary target include contributions from laboratory vibrations, laser phase noise inherent in the laser, electronic interferometer control fluctuations, electronic signal amplification noise, and shot noise in the detection process. These components determine the maximum sensitivity of the Fabry-Perot system and are present in all of the measurements.
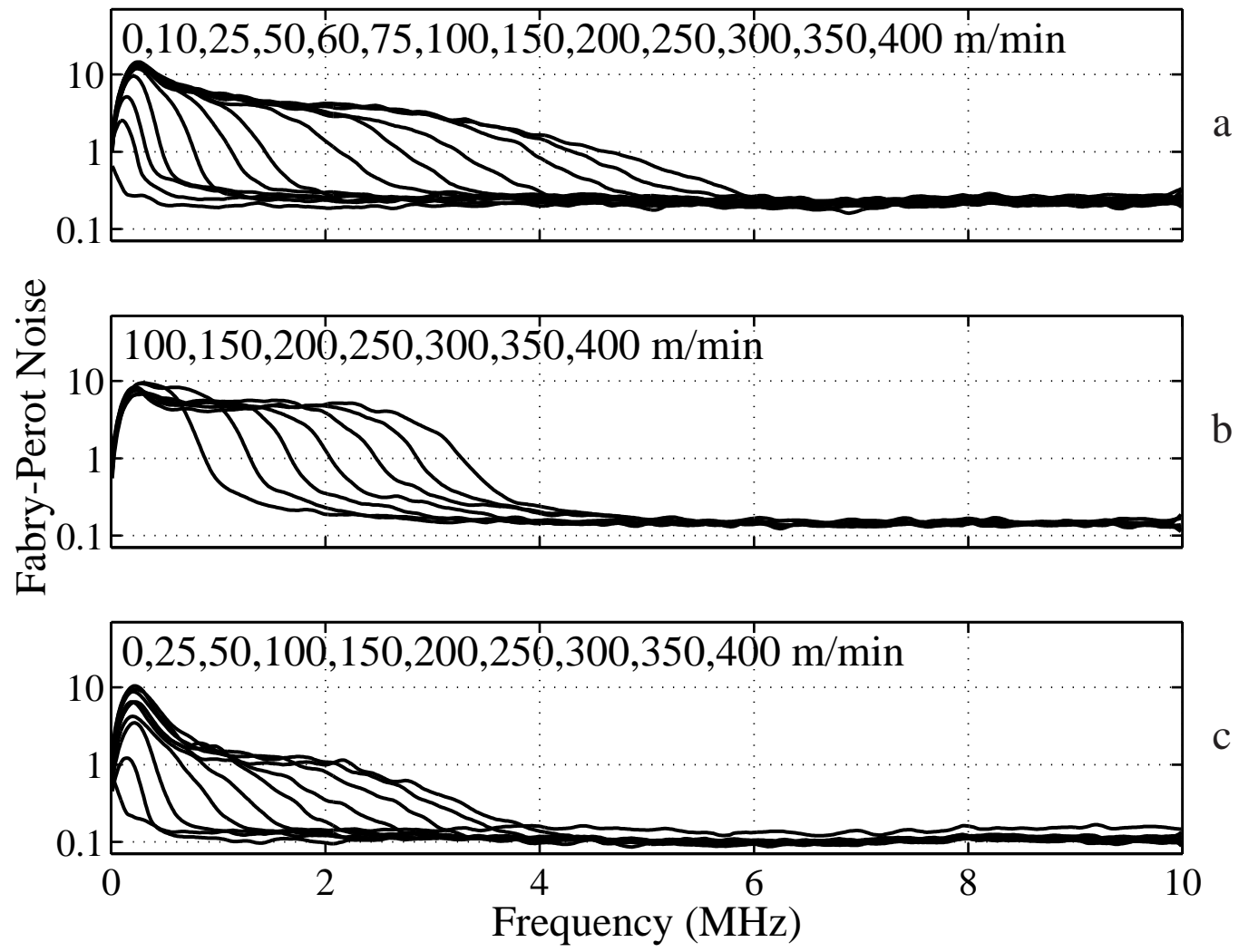

Figure 5. Noise spectra at various web simulator speeds for 3 situations, a) $0.04 \mathrm{~mm}$ and b) $0.26 \mathrm{~mm}$ detection spots on 59 \# raw stock paper, and c) $0.06 \mathrm{~mm}$ detection spot on the specular surface of the web simulator wheel. 


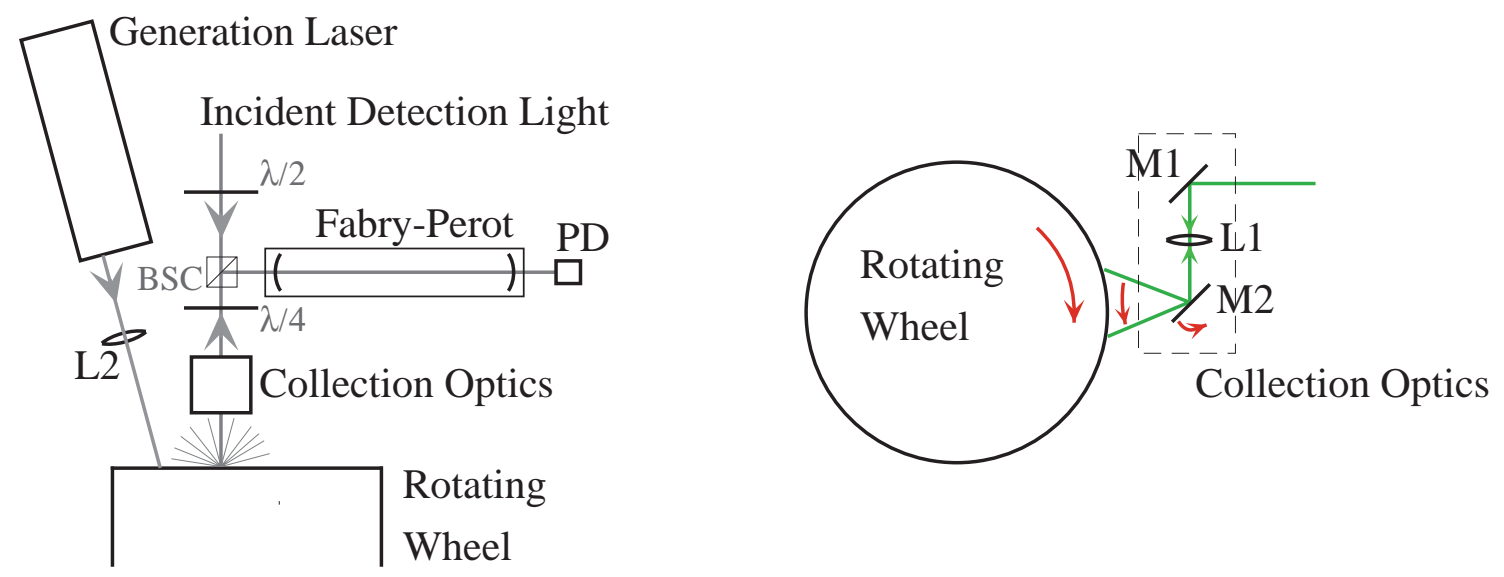

Figure 6. Optics setup to minimize speckle noise for moving sample. Mirror M1 is fixed while $\mathrm{M} 2$ rotates in sync with generation laser pulse.

The additional noise from the moving surface is attributed to a) flutter noise due to misalignment and vibrations in the web simulator, and b) speckle noise due to the moving speckle pattern at the detector. The flutter noise increases with web speed and accounts for the low frequency, web speed dependent component. The speckle noise has fixed maximum amplitude, since the optical phase shift is limited to modulo $2 \pi$. The frequency at which these speckles move across the detector aperture increases with web speed extending the bandwidth observed and accounts for the plateau component.

\section{$\underline{\text { Noise Avoidance }}$}

The speckle noise can be avoided, for the most part, by making measurements with the setup depicted in Figure 6. The tracking mirror M2 was rapidly swept through several degrees to match the speed of the detection spot to the speed of the paper at the time of the measurement. The polarized beam splitter cube BSC and wave plates $\lambda / 2$ and $\lambda / 4$ were added to provide coaxial illumination and detection beams during the sweep. Thus the same spot on the moving paper was illuminated during the measurement time and the

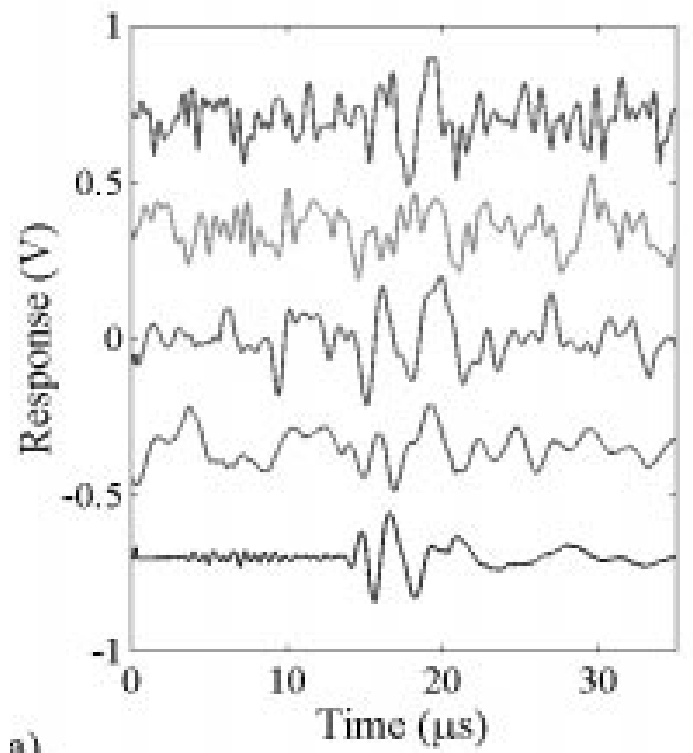

a)

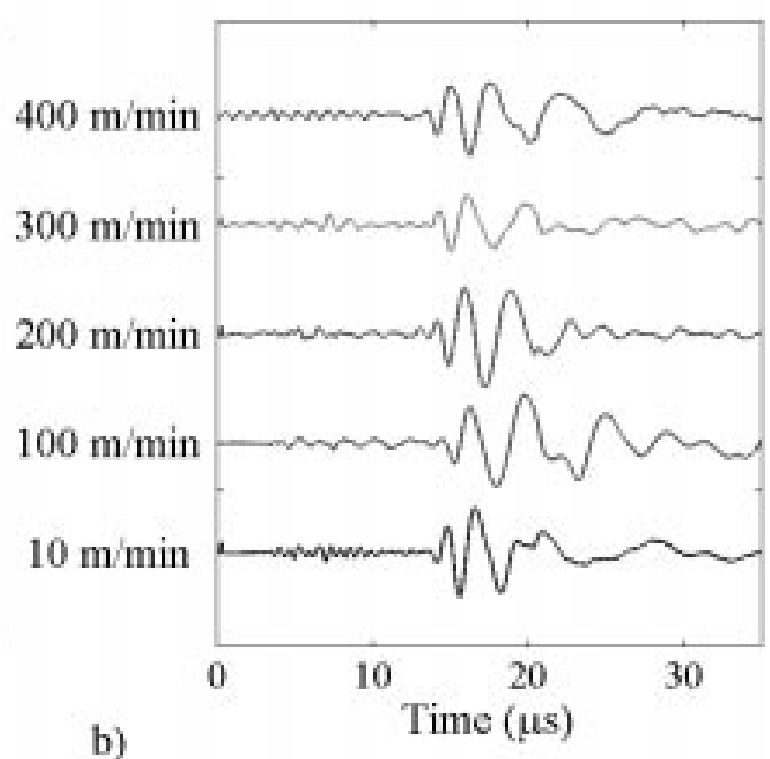

Figure 7. Comparison of waveforms detected a) without and b) with tracking for 59\# raw stock paper moving at $100,200,300$ and $400 \mathrm{~m} / \mathrm{min}$. The traces at $10 \mathrm{~m} / \mathrm{min}$ are without tracking, showing that it is only necessary to match the speed to within about $10 \mathrm{~m} / \mathrm{min}$. 
speckles were nominally stationary at the detector. Inclusion of the BSC sacrificed half of the light collected due to depolarization of the reflected light. Inclusion of M2 also limited the light collection efficiency, as it was kept small, about $12 \mathrm{~mm}$ across, to minimize its inertia. The trigger signals for the generation laser were derived from the position output of the control for M2, so the position of the generation spot could be selected relative to the position of the detection spot, and the ultrasonic measurement could be controlled and repeated.

Dramatic improvement is possible with tracking. The success of this reconfiguration is illustrated in Figure 7, which shows data recorded with M2 positioned $29 \mathrm{~mm}$ from the surface of the wheel. To compensate for the reduced light collection efficiency, the detection laser provided $2 \mathrm{~W}$ illumination at $532 \mathrm{~nm}$. Moving paper sustained much greater illumination than stationary paper due to the reduced time for local heating. The detection spot was focussed to about $0.5 \mathrm{~mm}$, which avoided damage to the paper. The plate waves were generated by an $11 \mathrm{~mJ}$ pulse at $1064 \mathrm{~nm}$ focussed to a roughly $1 \mathrm{~mm}$ diameter spot, displaced $8 \mathrm{~mm}$ laterally from the detection spot. All of the traces in Figure 7 are single shot, with frequency content limited to greater than $100 \mathrm{kHz}$ and less than $10 \mathrm{MHz}$. While there is little evidence in Figure $7 \mathrm{a}$ of the $\mathrm{A}_{0}$ waveform for the mirror not tracking, the $A_{0}$ waveform is clear at all speeds in Figure $7 \mathrm{~b}$ for the mirror tracking. Even the $S_{0}$ waveform can be identified at $3 \mu$ s in Figure $7 \mathrm{~b}$ for the paper moving at $300 \mathrm{~m} / \mathrm{min}$.

\section{CONCLUSION}

Ultrasonic measurements have been made on paper moving at up to $400 \mathrm{~m} / \mathrm{min}$ using a Fabry-Perot detector. The principle obstacle for measurements on a moving surface was speckle noise, due to the changing speckle pattern at the detector. This was overcome by moving the detection spot with the sample surface using a tracking mirror. The ultrasonic waveforms were recorded without averaging and with only minimal filtering, and showed the $S_{0}$ Lamb wave mode at up to $300 \mathrm{~m} / \mathrm{min}$, and the $A_{0}$ Lamb wave mode at up to $400 \mathrm{~m} / \mathrm{min}$. These measurements could be extended to paper mill speeds, $1600 \mathrm{~m} / \mathrm{min}$, with suitable changes in the tracking mirror.

\section{ACKNOWLEDGMENTS}

This work was sponsored by the U.S. Department of Energy, Office of Energy Efficiency and Renewable Energy, Office of Industrial Technologies under DOE Idaho Operations Office Contract DE-AC07-94ID13223.

\section{REFERENCES}

1. R. W. Mann, G. A. Baum and C. C. Habeger, Tappi 63, 163 (1980).

2. G. A. Baum, D. C. Brennan and C. C. Habeger, Tappi 64, 97 (1981).

3. C. C. Habeger and W. A. Wink, J. Appl. Polymer Sci.32, 4503 (1986).

4. P. H. Brodeur, M. S. Hall and C. Esworthy, J. Acoust. Soc. Am. 94, 2215 (1993).

5. M. Van Zummeran, D. Young, C. Habeger, G. Baum and R. Treleven, Ultrasonics 25, 288 (1987).

6. G. A. Baum, Appita 40, 288 (1985).

7. C. C. Habeger, M. Van Zummeran, W. A. Wink, B. M. Pankonin and R. S. Goodlin, Tappi 72, 171 (1989).

8. J.-P. Monchalin, IEEE Trans. UFFC 33, 485 (1986).

9. K. L. Telschow, J. B. Walter and G. V. Garcia, J. Appl. Phys. 68, 6077 (1990) 
10. M. A. Johnson, Y. H. Berthelot, P. H. Brodeur and L. A. Jacobs, Ultrasonics 34, 703 (1996).

11. J. C. Cheng and Y. H. Berthelot,J. Phys. D. 29, 1857 (1996).

12. P. H. Brodeur, M. A. Johnson, Y. H. Berthelot and J. P. Gerhardstein, J. Pulp and Paper Science 23, J238 (1997).

13. B. Pufahl, private communication, Jan 9, 1998. 